\title{
Primary chondrosarcoma of the thyroid cartilage: Surgery-based management of a rare case
}

\author{
GUO-HUA SUN ${ }^{1,2^{*}}$, YUAN-JIN WANG ${ }^{3 *}$, LI-LI GAO ${ }^{2,4^{*}}$, NING QU $^{1,2}$, \\ XIANG-MING $\mathrm{MU}^{3}$, RONG-LIANG SHI ${ }^{1,2}$ and QING-HAI JI ${ }^{1,2}$ \\ ${ }^{1}$ Department of Head and Neck Surgery, Fudan University Shanghai Cancer Center; ${ }^{2}$ Department of Oncology, \\ Shanghai Medical College, Fudan Universtiy, Shanghai 200032; ${ }^{3}$ Department of General Surgery, Yancheng \\ First People's Hospital, The Fourth Affiliated Hospital of Nantong Medical College, Yancheng, Jiangsu 224000; \\ ${ }^{4}$ Department of Pathology, Fudan University Shanghai Cancer Center, Shanghai 200032, P.R. China
}

Received May 19, 2016; Accepted September 8, 2016

DOI: $10.3892 / \mathrm{ol} .2016 .5257$

\begin{abstract}
In this study, we present a case of a 52-year-old male with a chondrosarcoma of the left lamina of the thyroid cartilage. Pre-operative evaluations detected typical calcifications and delineated the extent of the tumor. The patient underwent a total laryngectomy to ensure the complete resection of the tumor. The tumor was histopathologically found to consist of chondrocytes in a hyaline cartilage matrix. The patient's postoperative course has been successful apart from the permanent tracheostomy. Herien, we discuss the methods and rationales for the diagnosis and management of and recovery from this rare tumor, and also provide a review of the literature.
\end{abstract}

\section{Introduction}

Primary chondrosarcoma of the thyroid cartilage is a rare malignancy. Chondrosarcoma of the larynx is also uncommon, comprising only $1 \%$ of laryngeal malignancies (1), of which approximately $20 \%$ arise from the thyroid cartilage, the second most common site after the posterior lamina of the cricoid cartilage (2). The pathogenesis of chondrosarcoma of the thyroid cartilage is unknown, although a recent review suggested that several precipitating factors, such as multiple hereditary exostoses, Ollier's disease, chondromyxoid fibroma, and previous

Correspondence to: Professor Rong-Liang Shi or Professor Qing-Hai Ji, Department of Head and Neck Surgery, Fudan University Shanghai Cancer Center, 270 Dong An Road, Shanghai 200032, P.R. China

E-mail: shirongliang@126.com

E-mail: jiqinghai@shca.org.cn

*Contributed equally

Key words: imaging, diagnosis, histology, chondrosarcoma irradiation may contribute to its tumorigenesis (3). However, almost all reported cases originate from Europe or America.

An epidemiological study by Zhang et al indicated that in 2010,20,272 new cases of laryngeal cancer were diagnosed in China, with a crude incidence rate of 1.54/100,000 in 2010, and $0.66 \%$ of all new cancer cases (4). The age-standardized rate in China was shown to be $1.18 / 100,000$, and $1.20 / 100,000$ worldwide (4). Thus, the incidence of and mortality associated with laryngeal cancer is still relatively low in China. New cases of laryngeal chondrosarcoma, and clinical experience with its management, are extremely rare in China. Fudan University Shanghai Cancer Center is one of the largest specialized cancer hospitals in China, and its Department of Head and Neck Surgery is required to add any new information on the treatment and management of this tumor into the national literature database, in order to facilitate a more in depth discussion of these experiences at home and abroad. In this study, we present an example of a case of chondrosarcoma arising in the thyroid cartilage, and review the literature on this rare neoplasm in this location in order to provide surgeons with any new insight into its optimal management.

\section{Case report}

A 52-year-old male was admitted to the hospital (Fudan University Shanghai Cancer Center, Shanghai, China) in April 2015 for the evaluation of a firm non-tender left-sided neck mass that had reportedly become enlarged over a 2-year period. He had no history of neck pain, hoarseness, dysphagia, or dyspnea. An examination revealed a painless, fixed, rounded cervical lesion located in the left anterior triangle measuring $6.0 \times 5.0 \mathrm{~cm}$ in diameter, which moved on swallowing and adhered to the deep planes but showed no evidence of adenopathy. Ultrasonography revealed a large mass that extended to the left thyroid lobe; it was homogeneous, hypervascularized and solid. Both biological thyroid function tests and serum tumor markers were normal. Upon fibrolaryngoscopy, the mucosa of the right piriform sinus remained intact, but underwent slight medial displacement. No evidence of endolaryngeal involvement or neoplasm from other structural elements of the 

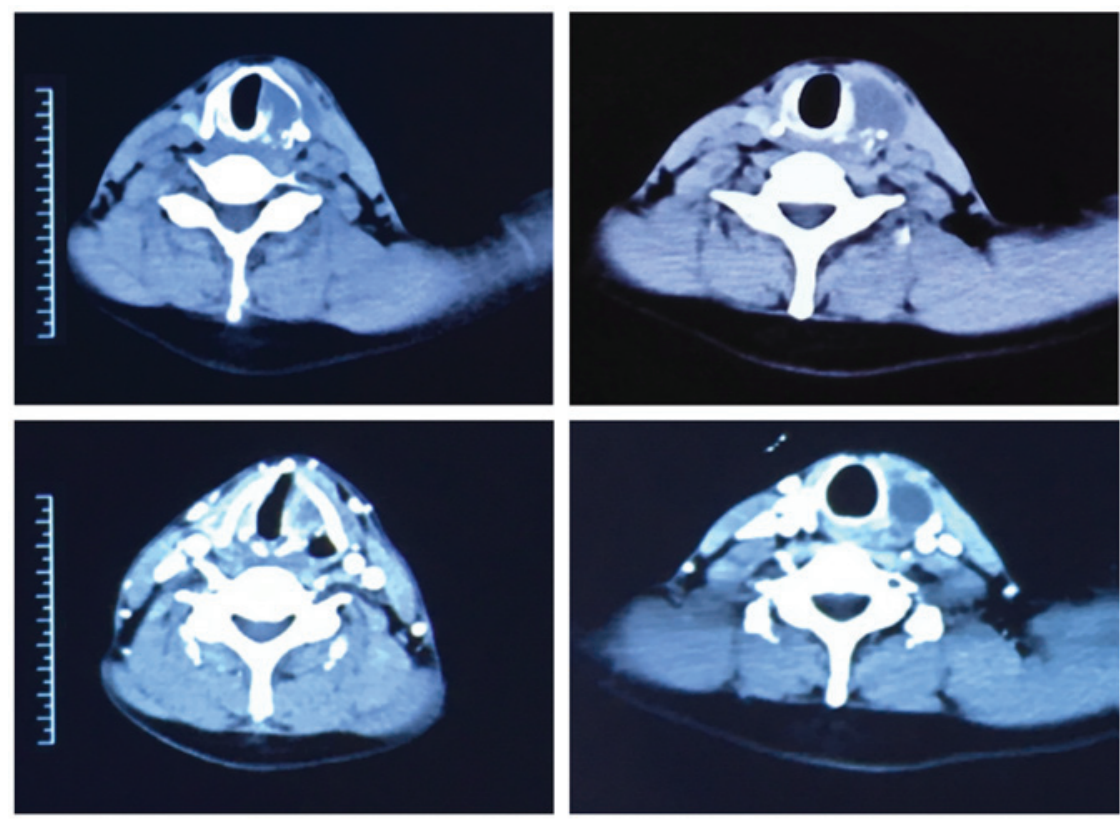

Figure 1. Plain and contrast-enhanced computed tomography axial scanning showing a large tumor mass in the left thyroid cartilage and characteristic multiple calcifications within the mass.
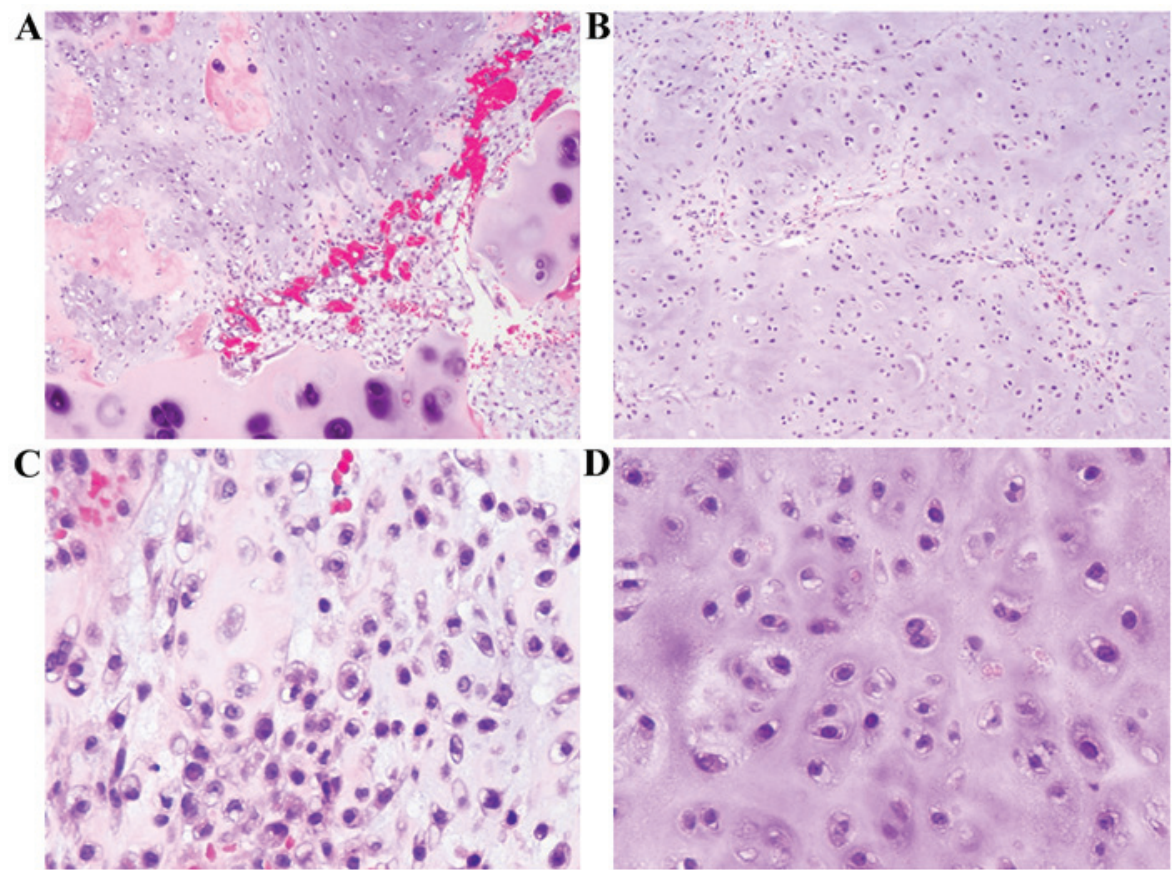

Figure 2. (A) Chondrosarcoma. Note the permeation of host bone [hematoxylin and eosin (H\&E) staining, x100 magnification]. (B) Conventional chondrosarcoma grade I, with scattered, relatively bland chondrocytes of various sizes and shapes in a hyaline cartilage matrix (H\&E staining, x100 magnification). (C) Focal areas of grade II chondrosarcoma demonstrate increased cellularity, and greater degrees of nuclear atypia and hyperchromasia, and larger nuclei (H\&E staining, $\mathrm{x} 400$ magnification). (D) Binucleation is osbserved (H\&E staining, $\mathrm{x} 400$ magnification).

larynx (e.g., cricoid cartilage, arytenoid cartilage) or the fixation of the vocal cords was observed.

Computed tomography (CT) is reported to be superior to plain films in showing the exact location and extent of the tumor. Moreover, CT scans document even minimal deposits of calcifications, which cannot be detected on conventional radiographs $(5,3)$. This patient's pre-operative $\mathrm{CT}$ scan revealed an expansive mass of $6.0 \times 5.0 \mathrm{~cm}$ in the posterior area of the left thyroid cartilage lamina without gross inflation or cortical breakage (Fig. 1). The mass extended adjacently to the left thyroid lobe, without involving the true vocal cord homo-laterally, the posterior commissure and arytenoid cartilage. Calcific foci with a popcorn-like appearance suggested a chondral origin.

Our diagnostic impression from the $\mathrm{CT}$ images and other evaluations was probable chondroma or chondrosarcoma (5). Pathological findings from a fine-needle aspirate also suggested 

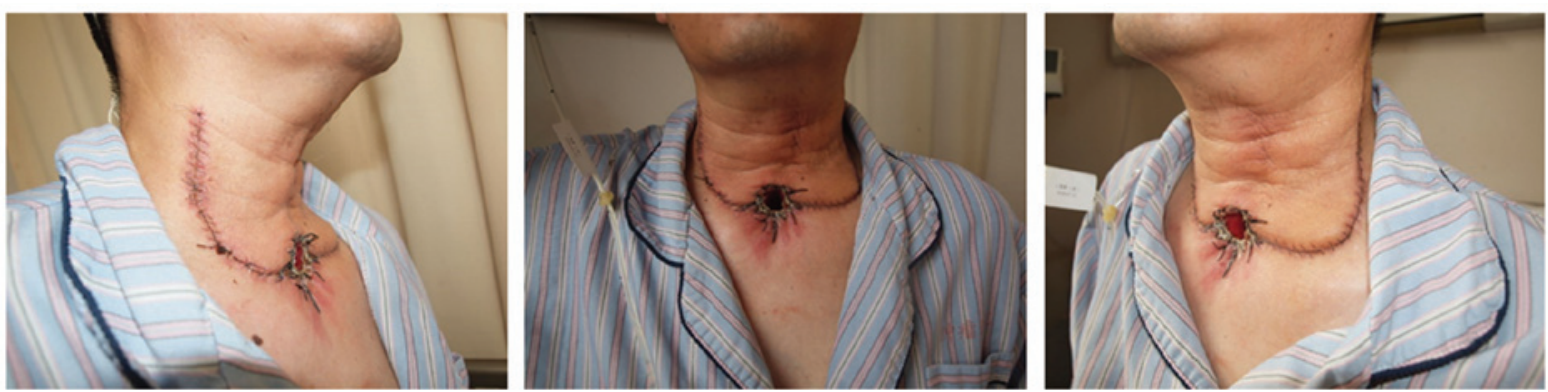

Figure 3. Post-operative recovery at 1 month.

chondroma or well-differentiated chondrosarcoma. The [F-18] fluorodeoxyglucose positron emission tomography is helpful in grading tumors, detecting metastasis and assessing local recurrence (6). The uptake value of 3.3 suggested a malignancy, but with good differentiation; 1.3 is generally considered the limit between benign and malignant lesions. It also showed no evidence of metastasis to the lung or bone.

As the primary tumor had spread out of the endolarynx by invading the thyroid cartilage, and as radiation or chemoradiation therapy were inadequate at initial treatment, the patient was recommended to undergo a total laryngectomy, left lobectomy of thyroid and a tracheotomy. An apron incision was outlined to incorporate the tracheostomy incision. The surgery itself was uneventful (7).

Histopathological examination of the resected specimen revealed areas of increased cellularity and a greater degree of cytological abnormality. Neoplastic cells formed clusters of irregular size and distribution (grade I, chondrosarcoma with myxoid components), with focal areas of grade II chondrosarcoma (Fig. 2).

Chondrosarcoma is considered to be insensitive to both radiation therapy (8) and chemotherapy. Thus, the patient was regularly followed-up without any adjuvant therapy, at a projected schedule of 1, 3, 6 and 12 months post-surgery, and every 6 months thereafter (Fig. 3). He is presently symptom-free without any sign of recurrence.

\section{Discussion}

Primary chondrosarcoma arising from the larynx is a rare disorder of unknown etiology, with a gradual development and a long clinical course (9). A chondrosarcoma that develops from the thyroid cartilage typically protrudes laterally and presents as a firm mass in the neck, but does not tend to grow into the airways or cause airway obstruction. Owing to this asymptomatic progression, these tumors are frequently in a locally advanced state at diagnosis. The pre-operative evaluation protocol includes a series of imaging examinations and cytology by needle biopsy $(1,10)$. Imaging modality is important for locating and delineating the primary tumor and regional metastases, but is unsatisfactory in distinguishing chondromas from chondrosarcomas $(5,11)$.

Some authors have suggested that $\mathrm{CT}$ is the imaging method of choice; it discloses a hypodense, well-defined image with calcifications inside, cartilage destruction and structure distortion. Others prefer magnetic resonance imaging (MRI) owing to its greater accuracy in distinguishing between tumor and other para-laryngeal tissues, with signal strength is low on T1 and high on T2, with a characteristic mosaic pattern $(6,12)$.

Unlike carcinomas in the neck area, pre-operative cytological biopsy may not distinguish neoplasms originating from the elastic cartilage from low-grade cervical sarcoma $(11,13)$, or between chondroma and low-grade malignancy.

The criteria for pathological diagnosis of chondrosarcoma include the presence of a number of cells with large, irregular and/or multiple nuclei, giant cartilage cells with large single or multiple nuclei and nuclei containing clumped chromatin upon histology (14), and are the basis for 3 grades of chondrosarcoma. Grade I is similar to a chondroma, $>2$ nuclei, no mitoses and some areas of calcification and actual bone tissue, and accounts for 70-80\% of cases; grade II involves an increased cell number, a low nuclear/cytoplasmic ratio and few mitoses; and grade III involves multinucleated cells, an increased nuclear/cytoplasmic ratio and a high number of mitoses (1). Notably, however, grade I (as in the present case) is histologically difficult to differentiate from chondroma, and requires combined clinical, radiological and histological signs to diagnose. In uncertain cases, lesions exceeding $2 \mathrm{~cm}$ are usually considered to be chondrosarcomas $(10,15)$.

Surgery is the treatment of choice, in order to excise all tumor extensions to negative margins, using either an external approach or endoscopy. However, cancer-specific recurrence is common, with a reported rate of $18-40 \%(1,6,8,16)$. The completeness of initial surgery is crucial to local disease control. In the present case, we performed a total laryngectomy in order to reduce the risk of local recurrence, having observed the wide extent of the primary tumor and partial high-grade (grade II) elements. Neither radiation therapy nor chemotherapy seems to be effective in eliminating subclinical metastasis postoperatively, particularly for low-grade, slow-growing tumors with few proliferating cells $(17,18)$. However, the long-term prognosis for this rare tumor improves with radical surgery, possibly due to its indolent nature.

A recent review of 'Survival in Chondrosarcoma,' based on the Surveillance, Epidemiology, and End Results (SEER) database, suggested that head and neck bone sarcomas commonly have a more favorable prognosis than axial tumors, which is partially explained by earlier detection, expeditious treatment and fewer metastases at presentation (19). Owing to the rarity of this tumor, our presentation is considered to be a literaturebased clinical case. It also provides validation and experience on its management from Eastern China and this may promote communication and cooperation on clinical decision-making, etiology, risk factors and therapeutic choices in the future. 


\section{Acknowledgements}

The present study was supported by funds from the National Science Foundation of China (grant nos. 81572622 and 81272934 awarded to Dr Qing-Hai Ji) and the Natural Science Foundation of Shanghai (grant no. 12JC1402802 awarded to Dr Qing-Hai Ji).

\section{References}

1. Polaczkiewicz D, Kochanowski B and Jakubiszyn J: Laryngeal chondrosarcoma. Otolaryngol Pol 51 (Suppl 25): 50-52, 1997 (In Polish).

2. Oudidi A, Hachimi $\mathrm{H}$ and El Alami MN: Laryngeal chondrosarcoma. Cancer Radiother 9: 343-346, 2005 (In French).

3. Potochny EM and Huber AR: Laryngeal chondrosarcoma. Head Neck Pathol 8: 114-116, 2014

4. Zhang SS, Xia QM, Zheng RS and Chen WQ: Laryngeal cancer incidence and mortality in China, 2010. J Cancer Res Ther 11 (Suppl 2): C143-C148, 2015.

5. Obenauer S, Kunze E, Fischer U, Schmidberger H and Grabbe E: Unusual chondrosarcoma of the larynx: CT findings. Eur Radiol 9: 1625-1628, 1999.

6. Rinaldo A, Howard DJ and Ferlito A: Laryngeal chondrosarcoma: a 24-year experience at the Royal National Throat, Nose and Ear Hospital. Acta Otolaryngol 120: 680-688, 2000.

7. Jabbour $\mathrm{N}$ and Tsue $\mathrm{T}$ : National operative case log growth charts in otolaryngology-head and neck surgery training. Otolaryngol Head Neck Surg 152: 73-79, 2015.

8. Domenech Campos E, Navarro-Conde P, Campos Dana JJ, Fontal Alvarez M, Zaragosí Castelló JM and Terradez Raro JJ: Advanced stage laryngeal chondrosarcoma. An Otorrinolaringol Ibero Am 29: 473-481, 2002 (In Spanish).
9. Eraso A, Lorusso GD and Palacios E: Laryngeal chondrosarcoma. Ear Nose Throat J 84: 402-403, 2005.

10. Hoffer ME, Pribitkin E, Keane WM and Atkins JP: Laryngeal chondrosarcoma: diagnosis and management. Ear Nose Throat J 71: 659-662, 1992.

11. Ibarrola C, Vargas J and de Agustín P: Laryngeal chondrosarcoma: fine needle aspiration (FNA) of an unusual tumour. Cytopathology 9: 130-134, 1998.

12. Chaturvedi A and Kane SV: Laryngeal chondrometaplasia: a great mimic of chondrosarcoma. Indian J Pathol Microbiol 50: 391-394, 2007.

13. Brown DH, Turnbull DI and Heeneman H: Laryngeal chondrosarcoma: gross pathological, histologic and electron microscopic characteristics. Can J Surg 28: 534-536, 1985.

14. Milanesi I: Histopathogenetic considerations on a case of laryngeal chondrosarcoma. Ann Laringol Otol Rinol Faringol 67: 767-776, 1968 (In Italian).

15. Pareja Martínez A, Alberola Toriol V, Severa Ferrándiz G and Infante Matarredona E: Chondrosarcoma of the thyroid cartilage. Presentation of a case and review of the literature. Acta Otorrinolaringol Esp 40: 373-376, 1989 (In Spanish)

16. Gierek T, Namysłowski G, Bielawska K and Myrcik H: Laryngeal chondrosarcoma. Otolaryngol Pol 35: 173-176, 1981 (In Polish).

17. Nicolai P, Ferlito A, Sasaki CT and Kirchner JA: Laryngeal chondrosarcoma: incidence, pathology, biological behavior, and treatment. Ann Otol Rhinol Laryngol 99: 515-523, 1990.

18. Ghalib SH, Warner ED and DeGowin EL: Laryngeal chondrosarcoma after thyroid irradiation. JAMA 210: 1762-1763, 1969.

19. Miller BJ: CORR Insights ${ }^{\circledR}$ : survival in mesenchymal chondrosarcoma varies based on age and tumor location: a survival analysis of the SEER Database. Clin Orthop Relat Res: Apr 5, 2016 (Epub ahead of print). 